\title{
On the limiting Stokes wave of extreme height in arbitrary water depth
}

\author{
Xiaoxu Zhong ${ }^{3}$ and Shijun Liao ${ }^{1,2,3, \dagger}$ \\ ${ }^{1}$ State Key Laboratory of Ocean Engineering, Shanghai 200240, China \\ ${ }^{2}$ Collaborative Innovative Center for Advanced Ship and Deep-Sea Exploration, Shanghai 200240, China \\ ${ }^{3}$ School of Naval Architecture, Ocean and Civil Engineering, Shanghai Jiao Tong University, \\ Shanghai 200240, China
}

(Received 30 August 2017; revised 26 December 2017; accepted 13 February 2018;

first published online 23 March 2018)

Both Schwartz (J. Fluid Mech., vol. 62 (3), 1974, pp. 553-578) and Cokelet (Phil. Trans. R. Soc. Lond., vol. 286 (1335), 1977, pp. 183-230) failed to gain convergent results for limiting Stokes waves in extremely shallow water by means of perturbation methods, even with the aid of extrapolation techniques such as the Padé approximant. In particular, it is extremely difficult for traditional analytic/numerical approaches to present the wave profile of limiting waves with a sharp crest of $120^{\circ}$ included angle first mentioned by Stokes in the 1880s. Thus, traditionally, different wave models are used for waves in different water depths. In this paper, by means of the homotopy analysis method (HAM), an analytic approximation method for highly nonlinear equations, we successfully gain convergent results (and especially the wave profiles) of the limiting Stokes waves with this kind of sharp crest in arbitrary water depth, even including solitary waves of extreme form in extremely shallow water, without using any extrapolation techniques. Therefore, in the frame of the HAM, the Stokes wave can be used as a unified theory for all kinds of waves, including periodic waves in deep and intermediate depths, cnoidal waves in shallow water and solitary waves in extremely shallow water.

Key words: waves/free-surface flows

\section{Introduction}

The two-dimensional steady progressive gravity wave is one of the most classic problems in fluid mechanics, which can be tracked back to Stokes (1847, 1880), and has been widely studied by a lot of researchers (Michell 1893; Nekrasov 1920; Yamada 1957; Yamada \& Shiotani 1968; Schwartz 1972; Byatt-Smith \& Longuet-Higgins 1976; Vanden-Broeck \& Schwartz 1979; Chen \& Saffman 1980; Olfe \& Rottman 1980; Schwartz \& Fenton 1982; Hunter \& Vanden-Broeck 1983; Sulem, Sulem \& Frisch 1983; Vanden-Broeck 1986; Fenton 1990; Klopman 1990;

$\dagger$ Email address for correspondence: sjliao@sjtu.edu.cn 
Karabut 1998; Dallaston \& Mccue 2010; Lushnikov 2016; Lushnikov, Dyachenko \& Silantyev 2017). Among analytic approaches for this problem, perturbation methods are used most frequently. Stokes $(1847,1880)$ proposed a perturbation approach using the first Fourier coefficient, $a_{1}$, as the perturbation quantity, and then showed that the highest free-surface wave (i.e. limiting wave, or extreme wave) in deep water would have a sharply pointed crest, enclosing a $120^{\circ}$ angle. Schwartz (1974) carried out this expansion for a deep-water wave to the order 70 and found that, as the wave height $H$ increases, the first Fourier coefficient $a_{1}$ first increases until it reaches a peak value, and then decreases. In other words, a single $a_{1}$ corresponds to two different wave heights for large enough wave height $H$. Thus, Stokes' expansion for deep-water waves is invalid for the limiting/extreme wave height.

Then Schwartz (1974) used a new expansion parameter $\epsilon=H / 2$ in his perturbation approach, and carried out the perturbation expansion to the 117th order in deep water and to the 48th order in general water depths, respectively. Utilizing the Padé approximants and Shanks's iterated $e_{1}$ transformations (Shanks 1954), Schwartz (1974) successfully obtained converged results for the ratio of water depth to wavelength $d / \lambda>0.05$. However, his method relies on extrapolation to obtain the dispersion relation for very high waves, since his perturbation series for the square of phase velocity, $c^{2}$, only converges well for wave heights shorter than $97 \%$ of the maximum. In addition, Schwartz (1974) found that accurate wave profiles cannot be obtained for very high waves even with the aid of the Padé approximant, so he added some standard terms to the crest to account for the remainder of the profile. Note that Schwartz (1974) ascribed the failure of his perturbation method in shallow water to round-off error.

A new perturbation quantity

$$
\epsilon=1-\frac{v_{\text {crest }}^{2}}{c_{0}^{2}},
$$

where $v_{\text {crest }}$ and $c_{0}$ are the fluid speed at the crest in the reference frame moving with the wave and the speed of waves of infinitesimal amplitude, respectively, was considered by Longuet-Higgins \& Fenton (1974). Using this perturbation quantity, Longuet-Higgins \& Fenton (1974) found that the series under the use of Padé approximants converges better than that using $\epsilon=H / 2$. Further, another expansion parameter

$$
\epsilon=1-\frac{v_{\text {crest }}^{2} v_{\text {trough }}^{2},}{c^{2} c_{0}^{2}},
$$

where $v_{\text {trough }}$ and $c$ denote the fluid speed at the trough and the phase speed in the inertial frame, respectively, was used by Longuet-Higgins (1975). The computational efficiency was drastically improved by using this perturbation quantity. In addition, Longuet-Higgins (1975) proposed an alternative expansion parameter

$$
\epsilon=1-\frac{v_{\text {crest }}^{2} v_{\text {trough }}^{2}}{c^{4}} .
$$

Using (1.3) as the perturbation quantity, Cokelet (1977) carried out the expansion to the 120th order, and obtained convergent results for Stokes waves with $d / \lambda>$ 0.0168. However, Cokelet (1977) pointed out that his method cannot give accurate 
wave profiles even in cases of $d / \lambda<0.11$. Furthermore, Dallaston \& Mccue (2010) reconsidered both Schwartz's (Schwartz 1974) and Cokelet's (Cokelet 1977) schemes, but with exact calculations so as to void any round-off error. However, they found that both the series expansions of Schwartz (1974) and Cokelet (1977) actually cannot provide precise estimates of the limiting wave properties in extremely shallow water.

Besides perturbation methods (Schwartz 1974; Cokelet 1977), a variety of numerical methods were proposed for the limiting Stokes wave. One common numerical method is to minimize the mean-squared error in the kinematic and dynamic free-surface boundary conditions. Chappelear (1961) expanded the velocity and the profile equation in Fourier series, and then used the method of least squares to determine the Fourier coefficients. Dean (1965) employed an analytical streamfunction expression with a series of unknown coefficients to describe the waves, and then used a numerical perturbation method to determine the unknown coefficients. Similarly, the numerical method was used by Williams (1981) to minimize the error in the surface boundary conditions over a series of evenly spaced points. However, a new crest term was supplemented to the integral equation in Williams' numerical method. Williams (1981) found that introducing this new term can greatly accelerate the convergence, i.e. the same level of accuracy can be reached by fewer Fourier coefficients. This method (Williams 1981) was a significant progress in numerics for Stokes' wave, since it is one of the few methods that are both free from extrapolation and can give accurate results. Unfortunately, this method (Williams 1981) still fails for extremely shallow water $d / \lambda<0.0168$.

Rienecker \& Fenton (1981) used a finite Fourier series to reduce the free-surface conditions to a set of nonlinear algebraic equations, and then used Newton's iteration method to solve these nonlinear equations. By means of this method, the equations are satisfied identically at a number of points on the surface. This method was further simplified by Fenton (1988) who numerically solved all the necessary partial derivatives. However, Fenton (1988) found that it is sometimes still necessary first to solve a sequence of lower waves and then to extrapolate forward in height steps to reach the desired height. Vanden-Broeck \& Schwartz (1979) proposed an efficient numerical scheme to solve the steep gravity wave. They first formulated the steep gravity waves as a system of integro-differential equations, and then used Newton's iteration technique to solve the coupled equations. Using this numerical method, accurate results can be obtained even in the case of $d / \lambda=0.008$. In addition, Vanden-Broeck \& Miloh (1995) employed series truncation methods, which use a refinement of Davies-Tulin's approximation (Davies 1951; Tulin 1983), to solve the steep gravity waves. By means of these methods, accurate numerical results can be obtained in the cases of $d / \lambda \geqslant 0.0168$. It should be emphasized that these schemes are easier to implement than the boundary integral equation methods (Hunter \& Vanden-Broeck 1983).

Besides the property of limiting Stokes wave with a included $120^{\circ}$ angle in the crest, the non-monotonicity of the speed and energy near the limiting wave height, first found by Longuet-Higgins (1975), also received wide attention from researchers. Longuet-Higgins \& Fox (1978) proposed a matching technique for gravity waves of almost extreme form, and then successfully confirmed the existence of branch points of order 1/2, as predicted by Grant (1973), and of turning points in the phase velocity as a function of wave height. In addition, the asymptotic solution of Longuet-Higgins \& Fox (1978) indicates that there is an infinite number of turning points in the dispersion relation, momentum and energy for the wave height very close to the maximum height. However, many methods generally only capture one or 
two of these turning points (Chandler \& Graham 1993), although three turning points are found by Dallaston \& Mccue (2010).

Note that understanding the characterization of the singularity structure of the Stokes wave, such as the locations and scalings of the singularities, is of great help in theory (Tanveer 1991; Crew \& Trinh 2016). Dyachenko, Lushnikov \& Korotkevich (2014, 2016) analysed the distance $d_{c}$ from the lowest singularity in the upper half-plane (i.e. the square-root branch point) to the real line which corresponds to the fluid free surface, and then suggested a power law scaling $d_{c} \propto\left(H_{\max }-H\right)^{3 / 2}$. Using this power law scaling, Dyachenko et al. $(2014,2016)$ presented an estimate $H_{\max } / \lambda \approx 0.1410633$ in deep water. Moreover, a square-root branch point is found by Lushnikov (2016) to be the only singularity in the physical (first) sheet of the Riemann surface for a non-limiting Stokes wave. Then an infinite number of square-root singularities are found in the infinite number of non-physical sheets of Riemann surface after crossing a branch cut of a square root into the second and subsequently higher sheets of the Riemann surface. Furthermore, Lushnikov (2016) conjectured that a non-limiting Stokes wave at the leading order consists of the infinite product of nested square-root singularities, and that on increasing the steepness of the Stokes wave to the extreme form, these nested square-root singularities will simultaneously approach the real line from different sheets of Riemann surface and finally form together a $2 / 3$ power law singularity of the limiting Stokes wave. This conjecture was well supported by high precision simulations. In addition, the slow decay of the Fourier coefficients is a challenging problem for numerical methods due to the existence of the singularities for a limiting/approximately limiting Stokes wave. In order to move all complex singularities away from the free surface, Lushnikov et al. (2017) introduced a free parameter into an auxiliary conformal mapping so as to allow for finer resolution near the crest of the wave. They found that the numerical convergence rate is dramatically improved by adapting the numerical grid near singularities.

Up to now, there are only few methods (Schwartz 1974; Cokelet 1977; VandenBroeck \& Schwartz 1979; Williams 1981) capable of solving the two-dimensional limiting (extreme) steady progressive wave in very shallow water. Besides, almost all analytic/numerical methods fail to give accurate results (especially for the wave profile) for limiting waves in extremely shallow water, such as $d / \lambda<0.005$. In addition, most analytic/numerical methods rely on extrapolation techniques, such as the Padé approximant, so as to accelerate the convergence and remove singularities that limit a series radius of convergence. So, an approach that can yield accurate results for the two-dimensional limiting (extreme) progressive gravity wave in arbitrary water depth without using any kind of extrapolation technique is of great value. This is the motivation of this paper.

In this paper, the limiting Stokes wave in arbitrary water depth is successfully solved by an analytic approximation method, namely the homotopy analysis method (HAM) (Liao 1992; Liao 1999, 2003, 2009, 2010, 2012; Van Gorder \& Vajravelu 2008; Kimiaeifar et al. 2011; Mastroberardino 2011; Vajravelu \& Van Gorder 2012; Sardanyés et al. 2015; Liao, Xu \& Stiassnie 2016; Liu, Xu \& Liao 2017, 2018; Zhong \& Liao 2017, 2018). Unlike perturbation methods (Schwartz 1974; Cokelet 1977), the HAM is independent of any small/large physical parameter. Especially, different from all analytic approximations, there is a so-called 'convergence-control parameter' $\hbar$ in the frame of the HAM, which has no physical meaning but provides a convenient way to guarantee the convergence of series solutions. For example, perturbation techniques are invalid for large deformation of a von Kármán plate, a 
famous classic problem in solid mechanics. However, Zhong \& Liao (2017, 2018) successfully applied the HAM to gain convergent series solutions even for extremely large deformation of a von Kármán plate. It is worthwhile mentioning that some mathematical theorems of convergence have been rigorously proved in the framework of the HAM (Liao 2012). For instance, it has been proved by Liao (2012) that the power series given by the HAM

$$
u(t)=\lim _{m \rightarrow+\infty} \sum_{n=0}^{m} \mu_{0}^{m, n}(\hbar)(-t)^{n},
$$

where

$$
\mu_{0}^{m, n}(\hbar)=(-\hbar)^{n} \sum_{k=0}^{m-n}\left(\begin{array}{c}
n-1+k \\
k
\end{array}\right)(1+\hbar)^{k},
$$

converges to $1 /(1+t)$ in the intervals:

$$
-1<t<-\frac{2}{\hbar}-1, \quad \text { when } \hbar<0,
$$

and

$$
-\frac{2}{\hbar}-1<t<-1, \quad \text { when } \hbar>0,
$$

respectively. So, the power series (1.4) converges to $1 /(1+t)$ either in the interval $(-1,+\infty)$ if $\hbar<0$ tend 0 , or in the interval $(-\infty,-1)$ if letting $\hbar>0$ tend to 0 , respectively. In other words, the power series (1.4) given by the HAM can converge to $1 /(1+t)$ in its entire definition interval (except the singularity $t=-1$ ) by properly choosing the so-called 'convergence-control parameter' $\hbar$. This is a good example to illustrate that the HAM can dramatically improve the convergence of a series by means of the so-called convergence-control parameter. By contrast, the traditional power series:

$$
\frac{1}{1+t} \sim 1-t+t^{2}-t^{3}+t^{4}-\cdots
$$

only converges in the interval $(-1,1)$. Thus, the HAM can indeed greatly enlarge the convergence interval of solution series by means of properly choosing the so-called 'convergence-control parameter' $\hbar$. Note that perturbation methods for many problems have been found to be a special case of the HAM when $\hbar=-1$, as illustrated by Zhong \& Liao (2017, 2018), and this well explains why perturbation results are often invalid in cases of high nonlinearity. In addition, the HAM provides us with great freedom to choose initial approximation so that iterations can be easily used to accelerate convergence in the framework of the HAM. Besides, a better initial guess can also modify the convergence of iteration, too. More importantly, something completely new/different has been successfully obtained by means of the HAM: the steady-state resonant waves were first predicted by the HAM in theory (Liao 2011; Xu et al. 2012; Liu \& Liao 2014) and then confirmed experimentally in a laboratory (Liu et al. 2015): all of these illustrate the novelty and potential of the HAM for highly nonlinear problems.

There exist two challenges for the traditional perturbation methods: (i) the series solutions diverge either when the water depth is rather small or when the wave height 
approaches the peak value; (ii) the computational efficiency is rather low when the terms of the Fourier coefficients are large. For the first challenge, it is found that the convergence of series solutions of the limiting Stokes wave can be guaranteed by means of choosing a proper convergence-control parameter $\hbar$ in the framework of the HAM. Note that, since there is a singularity exactly located at the crest for a Stokes wave of extreme form, considering enough terms of the Fourier series is inevitable if results of high precision are required. For the second challenge, we used an iterative HAM approach to greatly accelerate convergence of all unknown Fourier coefficients. Thus, by means of an iteration HAM approach with a properly chosen convergence-control parameter $\hbar$, accurate results in arbitrary water depth can be obtained efficiently. More importantly, since all Fourier coefficients obtained by the HAM are convergent, accurate wave profiles in very shallow water can be presented without using any kind of extrapolation technique. Compared with the perturbation methods (Schwartz 1974; Cokelet 1977), our HAM approach is simpler, easier to use and is valid across almost the whole range of physical parameters, as mentioned later in this paper. All of these demonstrate the superiority of the HAM over the perturbation method for this famous problem in fluid mechanics.

This paper is organized as follows. Fundamental equations are given in $\S 2$. Procedures of the HAM for the limiting Stokes wave problem are presented in $\S 3$. The limiting (extreme) Stokes wave in infinite depth is considered in $\S 4$. The limiting Stokes waves in finite depth are investigated in $\S 5$, with comparison to previous results (Laitone 1960; Fenton 1972; Schwartz 1974; Cokelet 1977; Williams 1981). Concluding remarks are given in $\S 6$.

\section{Mathematical description of the limiting Stokes wave}

Consider symmetrical, two-dimensional, periodic gravity waves propagating from right to left in a fluid with a horizontal bottom. The propagation of waves is only under the influence of gravity. Wave speed, $c$, is constant relative to an inertial frame. Assume that the fluid is inviscid and incompressible and that the motion is irrotational. Consider another reference frame moving with a wave crest. With respect to this frame, the motion is steady.

As shown in figure $1(a), \lambda, H, g$ represent the wavelength, the wave height and the gravity acceleration, respectively. Locate the $x$ axis at a distance $d$ above the bottom. Let the streamfunction $\Psi=0$ on the free surface, and $\Psi=-c d$ on the horizontal bottom. The Bernoulli condition on the free surface reads

$$
v \bar{v}+2 g y=K, \quad \Psi=0,
$$

where velocity $v=v_{x}-\mathrm{i} v_{y}$, the bar denotes the complex conjugation, and $K$ is an unknown constant.

As shown in figure 1, we map the interior of fluid motion 'ABODEA' in the physical ' $z$ ' plane into an annulus 'ABODEA' in the ' $\zeta$ ' plane according to the transformation:

$$
x+\mathrm{i} y=z(x, y)=z(\zeta)=\mathrm{i}\left[\ln \zeta+\sum_{j=1}^{+\infty} \frac{a_{j}}{j}\left(\zeta^{j}-\frac{r_{0}^{2 j}}{\zeta^{j}}\right)\right],
$$

where $\zeta=R \mathrm{e}^{\mathrm{i} \theta} ; R, r_{0}$ and $\theta$ represent the radius, inner radius and argument respectively; $a_{1}, a_{2}, \ldots, a_{j}, \ldots$ are unknown constant coefficients to be computed. The 
(a)

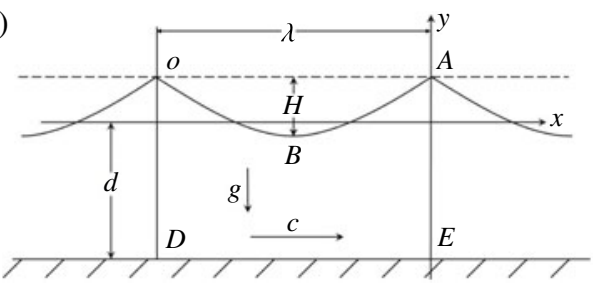

(b)

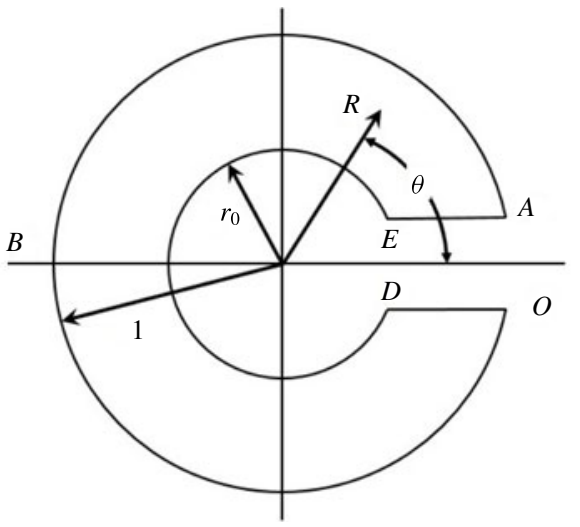

FIGURE 1. (a) $z$ plane, (b) $\zeta$ plane.

horizontal bottom $\Psi=-c d$ and the free surface $\Psi=0$ are then mapped onto the circles $R=r_{0}=\mathrm{e}^{-d}$ and $R=1$, respectively. Note that $r_{0}=0$ and $r_{0}=1$ correspond to the cases of infinite depth and infinite shallow water, respectively. The complex velocity potential $w$ can be expressed as

$$
w=\Phi+\mathrm{i} \Psi=\mathrm{i} c \ln \zeta=c \theta+\mathrm{i} c \ln R,
$$

where $\Phi$ represents velocity potential.

According to (2.2), we have

$$
\left.\begin{array}{l}
-x=\theta+\sum_{j=1}^{+\infty} \frac{a_{j}}{j}\left(R^{j}+\frac{r_{0}^{2 j}}{R^{j}}\right) \sin (j \theta), \\
y=\ln R+\sum_{j=1}^{+\infty} \frac{a_{j}}{j}\left(R^{j}-\frac{r_{0}^{2 j}}{R^{j}}\right) \cos (j \theta) .
\end{array}\right\}
$$

So, we have the wavelength

$$
\lambda=\left.x\right|_{R=1, \theta=0}-\left.x\right|_{R=1, \theta=2 \pi}=2 \pi,
$$

and the wave steepness

$$
\frac{H}{\lambda}=\frac{1}{2 \pi}\left(\left.y\right|_{R=1, \theta=0}-\left.y\right|_{R=1, \theta=\pi}\right)=\sum_{j=1}^{+\infty} \frac{a_{j}}{2 j \pi}\left(1-r_{0}^{2 j}\right)[1-\cos (j \pi)] .
$$

According to (2.3), the complex velocity $v$ reads

$$
v=\frac{\mathrm{d} w}{\mathrm{~d} z}=\frac{\mathrm{d} w}{\mathrm{~d} \zeta} \frac{\mathrm{d} \zeta}{\mathrm{d} z}=\frac{c}{f(\zeta)}
$$


where

$$
f(\zeta)=1+\sum_{j=1}^{+\infty} a_{j}\left(\zeta^{j}+\frac{r_{0}^{2 j}}{\zeta^{j}}\right) .
$$

Note that the velocity at the crest is zero for the highest wave. Using this restriction condition, equation (2.1) becomes

$$
v \bar{v}+2 g \int_{0}^{\theta} \operatorname{Im}\left[\frac{\mathrm{d} z}{\mathrm{~d} \zeta} \frac{\mathrm{d} \zeta}{\mathrm{d} \theta}\right] \mathrm{d} \theta=0, \quad \text { when } \Psi=0 .
$$

Substituting (2.2), (2.7), (2.8) into (2.9), we have the nonlinear algebraic equation

$$
\frac{2 g}{c^{2}} f \bar{f} \int_{0}^{\theta} \operatorname{Im}[f] \mathrm{d} \theta-1=0, \quad \text { at } R=1 .
$$

Theoretically, $a_{1}, a_{2}, \ldots, a_{j}, \ldots$ need to all be reserved to identically satisfy (2.10). However, we can only consider limited terms in practice. Thus, let us consider here the first $r$ Fourier coefficients $a_{1}, a_{2}, \ldots, a_{r}$, i.e. $f$ is approximated by

$$
f(\zeta) \approx a_{0}+\sum_{j=1}^{r} a_{j}\left(\zeta^{j}+\frac{r_{0}^{2 j}}{\zeta^{j}}\right), \quad a_{0}=1 .
$$

Substituting (2.11) into (2.10) and then equating the coefficients of $\cos (k \theta)$, where $k=$ $0,1,2, \ldots, r$, we obtain the following $(r+1)$ algebraic equations: (detailed derivation is shown in appendix A)

$$
c^{2}=g\left(2 j_{0} h_{0}+\sum_{n=1}^{r} j_{n} h_{n}\right),
$$

and

$$
\begin{aligned}
\mathcal{N}_{k} & {\left[a_{1}, a_{2}, \ldots, a_{r}\right] } \\
& =j_{0} h_{k}+j_{k} h_{0}+\frac{1}{2}\left(\sum_{n=1}^{k-1} j_{n} h_{k-n}+\sum_{n=1}^{r-k} j_{n} h_{n+k}+\sum_{n=1}^{r} j_{n+k} h_{n}\right)=0,
\end{aligned}
$$

where $\mathcal{N}_{k}(k=1,2, \ldots, r)$ denotes a nonlinear operator, with the following definitions:

$$
\begin{gathered}
h_{0}=\sum_{n_{1}=1}^{r} \frac{a_{n_{1}}\left(1-r_{0}^{2 n_{1}}\right)}{n_{1}}, \\
h_{n}=-\frac{a_{n}\left(1-r_{0}^{2 n}\right)}{n} \text { when } 1 \leqslant n \leqslant r, \\
j_{0}=1+\sum_{n_{1}=1}^{r} a_{n_{1}}^{2}\left(1+r_{0}^{4 n_{1}}\right), \\
j_{n}=2\left[\sum_{n_{1}=0}^{r-n}\left(1+r_{0}^{2 n+4 n_{1}}\right) a_{n_{1}} a_{n_{1}+n}+\sum_{n_{1}=1}^{n-1} r_{0}^{2 n-2 n_{1}} a_{n_{1}} a_{n-n_{1}}\right] \quad \text { when } 1 \leqslant n \leqslant r, \\
j_{n}=2 \sum_{n_{1}=n-r}^{r} r_{0}^{2 n-2 n_{1}} a_{n_{1}} a_{n-n_{1}} \text { when } r<n \leqslant 2 r .
\end{gathered}
$$


Then, the next step is to solve the nonlinear algebraic equations (2.13) for the $r$ unknown constant Fourier coefficients $a_{1}, a_{2}, \ldots, a_{r}$. Thereafter, the wave speed $c$ can be directly given by (2.12).

\section{The mathematical approach based on the HAM}

Let $a_{j, 0}$ denote the initial guess of $a_{j}(j=1,2, \ldots, r), \hbar$ a non-zero auxiliary parameter (called the convergence-control parameter) and $q \in[0,1]$ the embedding parameter for a homotopy, respectively. First of all, we construct a family of equations

$$
(1-q)\left[\Omega_{k}(q)-a_{k, 0}\right]=\hbar q \mathcal{N}_{k}\left[\Omega_{1}(q), \Omega_{2}(q), \ldots, \Omega_{r}(q)\right], \quad k=1,2, \ldots, r,
$$

where the nonlinear operators $\mathcal{N}_{1}, \mathcal{N}_{2}, \ldots, \mathcal{N}_{r}$ are defined by (2.13), and the unknown functions $\Omega_{1}(q), \Omega_{2}(q), \ldots, \Omega_{r}(q)$ correspond to the unknown constant Fourier coefficients $a_{1}, a_{2}, \ldots, a_{r}$, respectively, and $a_{k, 0}$ is the initial guess of $a_{k}$. Note that, in the frame of the HAM, we have great freedom to choose the initial guess $a_{k, 0}$ so that an iteration approach can be proposed based on this kind of freedom to greatly accelerate convergence, as mentioned later. More importantly, the so-called convergence-control parameter $\hbar$ can provide us with a simple way to guarantee the convergence of the solution series, as shown below.

Obviously, when $q=0$, equation (3.1) has the solution

$$
\Omega_{k}(0)=a_{k, 0}, \quad k=1,2, \ldots, r .
$$

When $q=1$, equation (3.1) is equivalent to the original equation (2.13), provided

$$
\Omega_{k}(1)=a_{k}, \quad k=1,2, \ldots, r .
$$

Therefore, as $q$ increases from 0 to 1 , the function $\Omega_{j}(q)$ varies (deforms) continuously from the known initial guess $a_{j, 0}$ to the unknown constant Fourier coefficient $a_{j}$, where $j=1,2, \ldots, r$. In the frame of the HAM, equation (3.1) is called the zeroth-order deformation equations. Obviously, according to (3.2), we have the Maclaurin series

$$
\Omega_{n}(q)=a_{n, 0}+\sum_{k=1}^{+\infty} a_{n, k} q^{k}, \quad n=1,2, \ldots, r
$$

where

$$
a_{n, k}=\mathcal{D}_{k}\left[\Omega_{n}(q)\right], \quad n=1,2, \ldots, r,
$$

in which

$$
\mathcal{D}_{k}[f]=\left.\frac{1}{k !} \frac{\partial^{k} f}{\partial q^{k}}\right|_{q=0}
$$

is called the $k$ th-order homotopy derivative of $f$. Note that, according to $(3.1), \Omega_{n}(q)$ and its series (3.4) are dependent upon the so-called convergence-control parameter $\hbar$. Assuming that $\hbar$ is properly chosen so that the Maclaurin series (3.4) exists and 
converges at $q=1$, then according to (3.3), we have the so-called homotopy-series solutions

$$
a_{n}=\sum_{k=0}^{+\infty} a_{n, k}, \quad n=1,2, \ldots, r .
$$

Substituting (3.4) into the zeroth-order deformation equations (3.1) and then equating the like powers of $q$, we have the so-called $m$ th-order deformation equations

$$
a_{k, m}-\chi_{m} a_{k, m-1}=\hbar \mathcal{D}_{m-1}\left[\mathcal{N}_{k}\right], \quad k=1,2, \ldots, r,
$$

where

$$
\begin{aligned}
\mathcal{D}_{i}\left[\mathcal{N}_{k}\right]= & \sum_{n_{2}=0}^{i}\left\{-\left[\frac{a_{k, i-n_{2}}\left(1-r_{0}^{2 k}\right)}{k}\right]\right. \\
& \times\left[1-\chi_{n_{2}+1}+\sum_{n_{1}=1}^{r} \sum_{n_{3}=0}^{n_{2}}\left(1+r_{0}^{4 n_{1}}\right) a_{n_{1}, n_{3}} a_{n_{1}, n_{2}-n_{3}}\right] \\
& +2\left[\sum_{n_{1}=1}^{r} \frac{a_{n_{1}, i-n_{2}}\left(1-r_{0}^{2 n_{1}}\right)}{n_{1}}\right]\left[\sum_{n_{1}=1}^{k-1} \sum_{n_{3}=0}^{n_{2}} r_{0}^{2 k-2 n_{1}} a_{n_{1}, n_{3}} a_{k-n_{1}, n_{2}-n_{3}}\right. \\
& \left.+\sum_{n_{1}=0}^{r-k} \sum_{n_{3}=0}^{n_{2}}\left(1+r_{0}^{2 k+4 n_{1}}\right) a_{n_{1}, n_{3}} a_{n_{1}+k, n_{2}-n_{3}}\right] \\
& -\sum_{n=1}^{k-1}\left[\frac{a_{k-n, i-n_{2}}\left(1-r_{0}^{2 k-2 n}\right)}{k-n}\right]\left[\sum_{n_{1}=1}^{n-1} \sum_{n_{3}=0}^{n_{2}} r_{0}^{2 n-2 n_{1}} a_{n_{1}, n_{3}} a_{n-n_{1}, n_{2}-n_{3}}\right. \\
& \left.+\sum_{n_{1}=0}^{r-n} \sum_{n_{3}=0}^{n_{2}}\left(1+r_{0}^{2 n+4 n_{1}}\right) a_{n_{1}, n_{3}} a_{n_{1}+n, n_{2}-n_{3}}\right] \\
& -\sum_{n=1}^{r-k}\left[\frac{a_{k+n, i-n_{2}}\left(1-r_{0}^{2 k+2 n^{2}}\right)}{k+n}\right]\left[\sum_{n_{1}=1}^{n-1} \sum_{n_{3}=0}^{n_{2}} r_{0}^{2 n-2 n_{1}} a_{n_{1}, n_{3}} a_{n-n_{1}, n_{2}-n_{3}}\right. \\
& \left.+\sum_{n_{1}=0}^{r-n} \sum_{n_{3}=0}^{n_{2}}\left(1+r_{0}^{2 n+4 n_{1}}\right) a_{n_{1}, n_{3}} a_{n_{1}+n, n_{2}-n_{3}}\right] \\
& -\sum_{n=1}^{r-k}\left[\frac{a_{n, i-n_{2}}\left(1-r_{0}^{2 n}\right)}{n}\right]\left[\sum_{n_{1}=1}^{n+k-1} \sum_{n_{3}=0}^{n_{2}} r_{0}^{2 n+2 k-2 n_{1}} a_{n_{1}, n_{3}} a_{n+k-n_{1}, n_{2}-n_{3}}\right. \\
& \left.+\sum_{n_{1}=0}^{r-n-k} \sum_{n_{3}=0}^{n_{2}}\left(1+r_{0}^{2 n+2 k+4 n_{1}}\right) a_{n_{1}, n_{3}} a_{n_{1}+n+k, n_{2}-n_{3}}\right] \\
& -\sum_{n=r-k+1}^{r}\left[\frac{a_{n, i-n_{2}}\left(1-r_{0}^{2 n}\right)}{n}\right] \\
& {\left.\left[\sum_{n_{1}=n+k-r}^{r} \sum_{n_{3}=0}^{n_{2}} r_{0}^{2 n+2 k-2 n_{1}} a_{n_{1}, n_{3}} a_{n+k-n_{1}, n_{2}-n_{3}}\right]\right\} } \\
&
\end{aligned}
$$


in which

$$
a_{0,0}=1, \quad a_{0, k}=0 \quad \text { when } k \geqslant 1,
$$

and

$$
\chi_{k}= \begin{cases}0 & \text { when } k \leqslant 1 \\ 1 & \text { when } k>1\end{cases}
$$

Note that, in the frame of the HAM, we have great freedom to choose the initial guesses $a_{1,0}, a_{2,0}, \ldots, a_{r, 0}$. So, we can simply choose

$$
a_{k, 0}=\frac{1}{k}, \quad k=1,2 \ldots, r .
$$

Then $a_{1, k}, a_{2, k}, \ldots, a_{r, k}$ can be obtained by (3.9) step by step, starting from $k=1$. The $n$ th-order homotopy approximations of $a_{1}, a_{2}, \ldots, a_{r}$ read

$$
\tilde{\Omega}_{i, n}=\sum_{k=0}^{n} a_{i, k}, \quad i=1,2 \ldots, r .
$$

Once $a_{1}, a_{2}, \ldots, a_{r}$ are determined, the wave speed $c$ can be given by (2.12).

In order to characterize the global error of our HAM approximation, we define the following squared residual error

$$
\mathcal{E}=\sum_{i=1}^{r}\left(\mathcal{N}_{i}\left[\tilde{\Omega}_{1}, \tilde{\Omega}_{2}, \ldots, \tilde{\Omega}_{r}\right]\right)^{2},
$$

where the nonlinear operators $\mathcal{N}_{1}, \mathcal{N}_{2}, \ldots, \mathcal{N}_{r}$ are defined by (2.13). Obviously, the smaller the $\mathcal{E}$, the more accurate the HAM approximation (3.13). Besides, it has been proved (Liao 2003, 2012) in general that a homotopy series converges to solution of original equations as long as all squared residual errors tend to zero. So, it is enough to check the squared residual error (3.14) only.

\section{The limiting Stokes wave in infinite depth}

To show the validity of our HAM approach mentioned above, we first of all give convergent series solution of the limiting (extreme) Stokes wave in infinite depth.

According to Liao (2003), the convergence of the homotopy-series solutions can be greatly accelerated by introducing the iteration technique, which uses the $n$ th-order homotopy approximation $\tilde{\Omega}_{1, n}, \tilde{\Omega}_{2, n}, \ldots, \tilde{\Omega}_{r, n}$ as new initial guesses $a_{1,0}, a_{2,0}, \ldots, a_{r, 0}$ for the next iteration, say, $a_{1,0}=\tilde{\Omega}_{1, n}, a_{2,0}=\tilde{\Omega}_{2, n}, \ldots, a_{r, 0}=\tilde{\Omega}_{r, n}$. This provides us with the $n$ th-order iteration of the HAM. According to our computation, both the HAM approach without iteration and the HAM-based iteration approach can yield convergent results, but the efficiency of the HAM-based iteration approach is much higher. In particular, the first-order HAM-based iteration approach has the highest efficiency. So, we use the first-order HAM-based iteration approach in all cases of this paper, if not specially mentioned.

Table 1 presents the results in the case of $r_{0}=0$, corresponding to infinite depth of water, given by the convergence-control parameter $\hbar=-0.2, r=100$ (i.e. one hundred truncated terms of Fourier series) and the initial guess (3.12). Note that the squared 


$\begin{array}{lccc}m, \text { iteration times } & \mathcal{E} & H / \lambda & (g \lambda) /\left(2 \pi c^{2}\right) \\ 20 & 1 \times 10^{-2} & 0.10623 & 1.0573 \\ 50 & 3 \times 10^{-3} & 0.15083 & 0.8153 \\ 100 & 5 \times 10^{-5} & 0.13846 & 0.8494 \\ 200 & 3 \times 10^{-9} & 0.13974 & 0.8422 \\ 300 & 3 \times 10^{-13} & 0.13973 & 0.8422 \\ 400 & 1 \times 10^{-17} & 0.13973 & 0.8422\end{array}$

TABLE 1. The squared residual error $\mathcal{E}$, wave steepness $H / \lambda$ and wave speed parameter $(g \lambda) /\left(2 \pi c^{2}\right)$ versus iteration times in the case of $r_{0}=0$, given by the first-order HAMbased iteration approach using $c_{0}=-0.2, r=100$ and the initial guess (3.12).

residual error $\mathcal{E}$ defined by (3.14) quickly decreases to the tiny level $10^{-17}$. This illustrates that all Fourier coefficients $a_{1}, a_{2}, \ldots, a_{100}$, given by our HAM approach, are convergent.

Figure 2 shows the homotopy approximation of the first Fourier coefficient, $a_{1}$, versus iteration times in the case of $r_{0}=0$, given by $r=100$ and the convergence-control parameter $\hbar=-0.4,-0.25,-0.1$. Note that $\hbar=-0.4$ leads to divergence of iteration, $\hbar=-0.25$ corresponds to a quickly convergent iteration but $\hbar=-0.1$ a slowly convergent iteration. Obviously, the optimal value of $\hbar$ corresponds to the fastest convergence, as pointed out by Liao (2010). It is found that convergent results can be obtained by our iteration HAM approach with arbitrary values of $\hbar \in[-0.27,0)$. So, the convergence-control parameter $\hbar$ indeed provides us with a simple way to guarantee convergence and to accelerate convergence. This clearly illustrates the important role of the convergence-control parameter $\hbar$ in the framework of the HAM.

Table 2 presents the convergence results in the case of $r_{0}=0$, given for different $r$. Note that the steepness of the limiting wave in infinite water depth tends to a fixed value $H / \lambda=0.14108$ when $r$ is large enough, say, $r>5000$. This is reasonable, since the precision of our results is controlled by $r$, i.e. the truncated number of the Fourier series (2.11). Note that Schwartz (1974) gave $H_{\max } / \lambda=0.14118$ but Dyachenko et al. (2016) gave $H_{\max } / \lambda=0.141058$ for a limiting wave in deep water, with $0.071 \%$ and $0.016 \%$ relative errors compared to our results, respectively. It should be emphasized that, by means of the HAM, convergent results of all Fourier coefficients $a_{j}$ can be obtained. This distinguishes the HAM from other methods.

It is found that the high-order Fourier coefficients $a_{j}$ drop rather slowly (e.g. $a_{1}=$ $0.29223, a_{100}=0.01576, a_{500}=0.005415, a_{1000}=0.003739, a_{3000}=0.002905, a_{5000}=$ $0.002759)$. We have $H / \lambda=0.14085$ even by means of $r=500$, and have the more accurate result $H / \lambda=0.14108$ by $r>5000$. All of these indicate that $f$ defined by (2.8) converges slowly indeed. However, it should be emphasized that, whatever $r$ we choose, convergent values of all Fourier coefficients $a_{j}$ can be directly obtained by our iterative HAM approach without using any extrapolation or Padé approximant techniques.

Figure 3 shows the comparison of limiting wave profiles given by Schwartz (1974), Dyachenko et al. (2016) and our HAM approach. The agreement between them is satisfactory. This indicates the validity of our HAM-based approach. Our limiting wave profile has a sharply pointed crest with an enclosing angle $119.3^{\circ}$, which is very close to the theoretical value $120^{\circ}$. However, Schwartz (1974) mentioned that "while the method of Páde fractions yields accurate profiles for wave heights somewhat short 


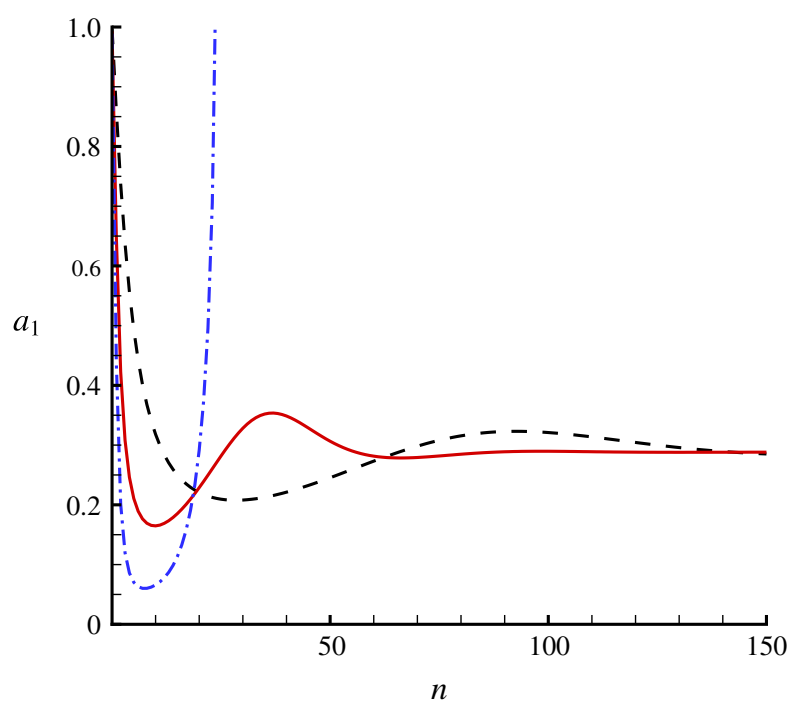

FigurE 2. (Colour online) The first Fourier coefficient, $a_{1}$, versus number of iterations, $n$, in the case of $r_{0}=0$, given by the first-order HAM-based iteration approach using $r=100$ and the convergence-control parameter $\hbar=-0.1,-0.25,-0.4$.,$--- \hbar=-0.1$; $\longrightarrow, \hbar=-0.25 ;-\cdots, \hbar=-0.4$.

$\begin{array}{lcc}r & H / \lambda & (g \lambda) /\left(2 \pi c^{2}\right) \\ 50 & 0.13926 & 0.8391 \\ 500 & 0.14085 & 0.8397 \\ 1000 & 0.14102 & 0.8388 \\ 2000 & 0.14107 & 0.8383 \\ 3000 & 0.14108 & 0.8382 \\ 4000 & 0.14109 & 0.8382 \\ 5000 & 0.14108 & 0.8381 \\ 6000 & 0.14108 & 0.8381\end{array}$

TABLE 2. Wave steepness $H / \lambda$ and wave speed parameter $(g \lambda) /\left(2 \pi c^{2}\right)$ versus truncated terms $r$ in the case of $r_{0}=0$ (in infinite depth), given by the first-order HAM-based iteration approach using $c_{0}=-0.2$.

of the maximum, it is insufficient for the description of very high waves', and that 'Páde fractions do not converge well in the immediate neighbourhood of branch-points; moreover, only the first few coefficients $a_{j}$, can be determined with acceptable accuracy'. Thus, Schwartz (1974) had to use the so-called 'series completion method' to gain a satisfactory wave profile. By contrast, using the HAM, convergent results of all Fourier coefficients $a_{j}(j=1,2,3, \ldots, r)$ and the convergent wave profile for the limiting wave can be obtained without using any extrapolation techniques such as the Padé technique, the series completion method and so on. This illustrates that the HAM-based approach is superior to perturbation methods (Schwartz 1974; Cokelet 1977). This is mainly because, unlike perturbation methods, the HAM provides us with a convenient way (through the so-called convergence-control parameter $\hbar$ ) to guarantee the convergence of solution series. 


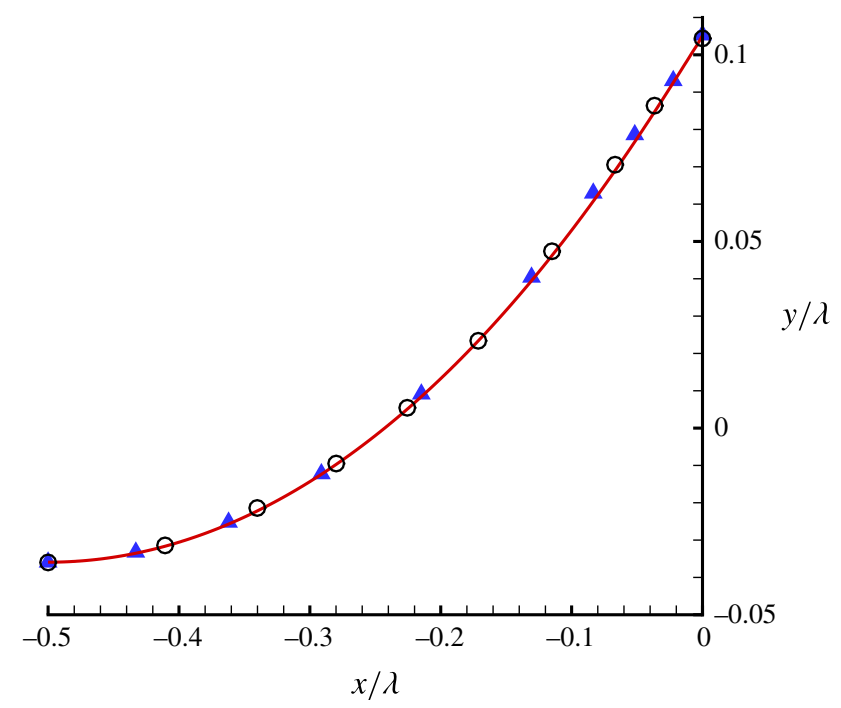

FIgURE 3. (Colour online) Wave profiles in the case of $r_{0}=0$. —, given by the first-order HAM-based iteration approach; $\bigcirc$, given by the numerical approach of Dyachenko et al. (2016); $\boldsymbol{\Delta}$, given by Schwartz's perturbation method with the aid of a so-called series completion method (Schwartz 1974).

\section{The limiting Stokes wave in finite depth}

Note that $r_{0}=0$ and $r_{0}=1$ correspond to the cases of infinite depth and infinitely shallow water, respectively. Without loss of generality, let us first consider the case of $r_{0}=0.05$. In the frame of the HAM, we have great freedom to choose the initial guesses of $a_{1}, a_{2}, \ldots, a_{r}$. Considering the continuous variation of $a_{1}, a_{2}, \ldots, a_{r}$ as $r_{0}$ increases from 0 to 1 , the convergent values of $a_{1}, a_{2}, \ldots, a_{5000}$ in the case of $r_{0}=0$, obviously, are much better than (3.12) as the initial guess for the case of $r_{0}=0.05$. In other words, if we have obtained the convergent results of $a_{1}, a_{2}, \ldots, a_{5000}$ in the case of $r_{0}=0$, then it is better to take

$$
a_{k, 0}= \begin{cases}a_{k} & \text { when } 1 \leqslant k \leqslant 5000, \\ a_{5000} & \text { when } k>5000,\end{cases}
$$

as the initial guesses of $a_{1}, a_{2}, \ldots, a_{r}$ in the case of $r_{0}=0.05$.

It is found that, in the case of $r_{0}=0.05$, the optimal convergence-control parameter $\hbar$ is approximately -0.2 if the initial guess (3.12) for $r_{0}=0$ is taken, and 400 iterations are required to gain convergent results $H / \lambda=0.14026$ and $(g \lambda) /\left(2 \pi c^{2}\right)=0.8421$, as shown in table 3. However, if we take the initial guess (5.1), the optimal convergent-control parameter $\hbar$ becomes -1.2 , and we obtain the same convergent results $H / \lambda=0.14026$ and $(g \lambda) /\left(2 \pi c^{2}\right)=0.8421$ with only thirty iterations, as shown in table 4 . Thus, the computational efficiency by means of the initial guess (5.1) is approximately 13 times higher than that by (3.12). This illustrates that our iterative HAM approach with the optimal convergence-control parameter $\hbar$ can indeed greatly accelerate the convergence.

Similarly, the convergent results in arbitrary water depth are successfully obtained by means of the above-mentioned strategy, as shown in table 5. Note that Liao (2010) suggested a general approach to gain an optimal convergence-control parameter in 


$\begin{array}{lcc}m, \text { iteration times } & H / \lambda & (g \lambda) /\left(2 \pi c^{2}\right) \\ 10 & 0.18670 & 0.5663 \\ 50 & 0.13681 & 0.9099 \\ 100 & 0.13976 & 0.8455 \\ 200 & 0.14033 & 0.8416 \\ 300 & 0.14023 & 0.8421 \\ 400 & 0.14026 & 0.8421 \\ 500 & 0.14026 & 0.8421\end{array}$

TABLE 3. Wave steepness $H / \lambda$ and wave speed parameter $(g \lambda) /\left(2 \pi c^{2}\right)$ versus iterations, $m$, in the case of $r_{0}=0.05$, given by the first-order HAM-based iterative approach using the convergence-control parameter $c_{0}=-0.2$, the truncated terms $r=5500$ and the initial guess (3.12).

$\begin{array}{lcc}m, \text { iteration times } & H / \lambda & (g \lambda) /\left(2 \pi c^{2}\right) \\ 10 & 0.14018 & 0.8423 \\ 20 & 0.14024 & 0.8422 \\ 30 & 0.14026 & 0.8421 \\ 40 & 0.14026 & 0.8421 \\ 50 & 0.14026 & 0.8421\end{array}$

TABLE 4. Wave steepness $H / \lambda$ and wave speed parameter $(g \lambda) /\left(2 \pi c^{2}\right)$ versus iterations, $m$, in the case of $r_{0}=0.05$, given by the first-order HAM-based iteration approach using the convergence-control parameter $c_{0}=-1.2$, the truncated terms $r=5500$ and the initial guesses (5.1).

the framework of the HAM. According to our computation, the interval of $\hbar$, which guarantees the convergence of iteration, becomes larger with the increase of $r_{0}$. It is found that, in the case of $0.05 k<r_{0} \leqslant 0.05(k+1)$, where $0 \leqslant k \leqslant 19$ is a natural number, the corresponding optimal convergence-control parameter $\hbar$ can be expressed by the following empirical formula

$$
\hbar=-1.2-\frac{k^{3}}{2000}, \quad 0 \leqslant k \leqslant 19,
$$

if we use the known convergent Fourier coefficients $a_{j}$ in the case of $r_{0}=0.05 k$ as the initial guess. Note that a convergence-control parameter $\hbar$ closer to 0 represents a slower convergence of solutions, i.e. a lower efficiency of computation, as shown in figure 2. Thus, the convergence-control parameter $\hbar$ provides us with a convenient way not only to guarantee the convergence of series solutions but also to improve the computational efficiency. It is found that, for all cases considered in table 5 , a few hundred iterations are enough to gain convergent results for all Fourier coefficients $a_{j}$.

In the case of extremely shallow water, a very large number of Fourier coefficients are needed to present the limiting wave with the sharp crest. Table 6 presents the convergent results given for different values of $r$ in the case of $r_{0}=0.99$. It is found that $r=50000$ can give the fixed results $H / \lambda=1.3281 \times 10^{-3}$ and $(g \lambda) /\left(2 \pi c^{2}\right)=60.175$ in the case of $r_{0}=0.99$. This indicates that our iterative HAM approach can indeed give convergent results for the limiting Stokes waves even in extremely shallow water. Note that, in case of $r=50000$, we must solve a set of 


$\begin{array}{lccccc}r_{0} & r & d / \lambda & H / \lambda & H / d & (g \lambda) /\left(2 \pi c^{2}\right) \\ 0 & 5000 & \infty & 1.4108 \times 10^{-1} & 0 & 0.8381 \\ 0.05 & 5500 & 4.77 \times 10^{-1} & 1.4026 \times 10^{-1} & 0.2942 & 0.8421 \\ 0.10 & 6000 & 3.66 \times 10^{-1} & 1.3782 \times 10^{-1} & 0.3761 & 0.8540 \\ 0.15 & 6500 & 3.02 \times 10^{-1} & 1.3386 \times 10^{-1} & 0.4433 & 0.8739 \\ 0.20 & 7000 & 2.56 \times 10^{-1} & 1.2851 \times 10^{-1} & 0.5017 & 0.9022 \\ 0.25 & 7500 & 2.21 \times 10^{-1} & 1.2197 \times 10^{-1} & 0.5528 & 0.9395 \\ 0.30 & 8000 & 1.92 \times 10^{-1} & 1.1446 \times 10^{-1} & 0.5973 & 0.9864 \\ 0.35 & 8500 & 1.67 \times 10^{-1} & 1.0618 \times 10^{-1} & 0.6355 & 1.0442 \\ 0.40 & 9000 & 1.46 \times 10^{-1} & 9.7388 \times 10^{-2} & 0.6678 & 1.1145 \\ 0.45 & 9500 & 1.27 \times 10^{-1} & 8.8289 \times 10^{-2} & 0.6947 & 1.2001 \\ 0.50 & 10000 & 1.10 \times 10^{-1} & 7.9084 \times 10^{-2} & 0.7169 & 1.3048 \\ 0.55 & 10500 & 9.51 \times 10^{-2} & 6.9943 \times 10^{-2} & 0.7351 & 1.4344 \\ 0.60 & 11000 & 8.13 \times 10^{-2} & 6.0995 \times 10^{-2} & 0.7502 & 1.5977 \\ 0.65 & 11500 & 6.86 \times 10^{-2} & 5.2327 \times 10^{-2} & 0.7632 & 1.8091 \\ 0.70 & 12000 & 5.68 \times 10^{-2} & 4.3983 \times 10^{-2} & 0.7748 & 2.0922 \\ 0.75 & 14000 & 4.58 \times 10^{-2} & 3.5968 \times 10^{-2} & 0.7856 & 2.4898 \\ 0.80 & 16000 & 3.55 \times 10^{-2} & 2.8263 \times 10^{-2} & 0.7958 & 3.0876 \\ 0.85 & 18000 & 2.59 \times 10^{-2} & 2.0840 \times 10^{-2} & 0.8057 & 4.0856 \\ 0.90 & 22000 & 1.68 \times 10^{-2} & 1.3670 \times 10^{-2} & 0.8152 & 6.0838 \\ 0.95 & 28000 & 8.16 \times 10^{-3} & 6.7292 \times 10^{-3} & 0.8243 & 12.084 \\ 0.97 & 37000 & 4.85 \times 10^{-3} & 4.0128 \times 10^{-3} & 0.8278 & 20.087 \\ 0.99 & 50000 & 1.60 \times 10^{-3} & 1.3281 \times 10^{-3} & 0.8303 & 60.175\end{array}$

TABLE 5. Results for a variety of water depths, given by the first-order HAM-based iteration approach.

50000 coupled, highly nonlinear algebraic equations! Fortunately, this is possible nowadays by means of a supercomputer such as $\mathrm{TH}-2$ at National Supercomputer Centre in Guangzhou, China. Finally, it should be emphasized that all of these convergent results are obtained directly, without using any extrapolation and Padé approximant techniques.

Stokes (1880) gave a famous conjecture that the limiting wave (with extreme height) should have a sharp crest with an included angle of $120^{\circ}$. Approximately one hundred years later, this conjecture was independently proved in mathematics by Amick, Fraenkel \& Toland (1982) and Plotnikov (2002) for Stokes waves in an arbitrary depth of water. However, to the best of our knowledge, the detailed wave profiles for the limiting Stokes wave in extremely shallow water have not been reported. Table 7 presents the included crest angles of the limiting Stokes wave in a variety of water depths, given by our iteration HAM approach. All of the included crest angles in different depths given by the HAM are very close to the theoretical value $120^{\circ}$. The wave profiles for a variety of water depths given by the HAM are shown in figure 4 . Note that the high-order Fourier coefficients $a_{j}$ play an important role in correctly describing the wave profile, especially the wave crest. For instance, although Cokelet's perturbation method (Cokelet 1977) can give $H / d$ with acceptable accuracy for $r_{0}<0.9$, it fails to give an accurate wave profile even for $r_{0}>0.5$. Fortunately, the HAM can always yield convergent results for all Fourier coefficients by means of choosing a proper convergence-control parameter $\hbar$. This once again illustrates the superiority of the HAM over other methods (Schwartz 1974; Cokelet 1977). 


$\begin{array}{lcc}r & H / \lambda & (g \lambda) /\left(2 \pi c^{2}\right) \\ 21000 & 1.3229 \times 10^{-3} & 60.410 \\ 28000 & 1.3251 \times 10^{-3} & 60.312 \\ 35000 & 1.3264 \times 10^{-3} & 60.249 \\ 40000 & 1.3272 \times 10^{-3} & 60.214 \\ 50000 & 1.3281 \times 10^{-3} & 60.175 \\ 55000 & 1.3281 \times 10^{-3} & 60.175\end{array}$

TABLE 6. Wave steepness $H / \lambda$ and wave speed parameter $(g \lambda) /\left(2 \pi c^{2}\right)$ versus truncated terms $r$ in the case of $r_{0}=0.99$, given by the first-order HAM-based iteration approach.

$\begin{array}{lc}r_{0} & \text { Included crest angle (deg.) } \\ 0 & 119.3 \\ 0.3 & 119.2 \\ 0.6 & 119.4 \\ 0.9 & 120.2 \\ 0.99 & 119.2\end{array}$

TABLE 7. Included crest angles in a variety of depths, given by the first-order HAM-based iteration approach.

According to the convergent results given by the iterative HAM approach, we have the fitted formulas of $H / d$ versus $c^{2} /(g d)$ and $\lambda / d$ :

$$
\begin{aligned}
\frac{H}{d} & =0.58557 \frac{c^{2}}{g d}+0.62667\left(\frac{c^{2}}{g d}\right)^{2}-0.73410\left(\frac{c^{2}}{g d}\right)^{3}+0.19634\left(\frac{c^{2}}{g d}\right)^{4}, \\
\frac{H}{d} & =\frac{0.14109 \frac{\lambda}{d}+0.00804\left(\frac{\lambda}{d}\right)^{2}+0.00949\left(\frac{\lambda}{d}\right)^{3}}{1+0.09671 \frac{\lambda}{d}+0.02695\left(\frac{\lambda}{d}\right)^{2}+0.01139\left(\frac{\lambda}{d}\right)^{3}},
\end{aligned}
$$

which agree quite well with our HAM results, as shown in figure 5.

Let us make some comparisons of the limiting Stokes waves given by different analytic/numerical methods. Table 8 presents the comparison of limiting wave steepness for a variety of depths. The results of Schwartz (1974) are accurate only for $r_{0}<0.7$; the results of Cokelet (1977) are accurate only for $r_{0} \leqslant 0.8$; the results of Williams (1981) are of high accuracy for $r_{0} \leqslant 0.9$, although they are slightly smaller. However, all of these methods fail to give convergent result for $r_{0}>0.9$, i.e. extremely shallow water. Fortunately, the HAM can give convergent results for the limiting waves for almost arbitrary depth.

Figure 6 shows the comparison of the limiting wave steepness $H / \lambda$, given by Schwartz (1974), Williams (1981) and the HAM approach mentioned in this paper. It is found that the perturbation method (Schwartz 1974) is only valid for $r_{0} \in[0,0.7]$ even with the aid of extrapolation and Padé approximant techniques; Williams' numerical method (Williams 1981) is only valid for $r_{0} \in[0,0.9]$. However, the HAM can give accurate convergent results even for $r_{0} \in[0,0.99]$.

Figure 7 shows the comparison of $H / d$ versus the squared Froude number, $c^{2} /(g d)$. It is found that Cokelet's perturbation method (Cokelet 1977) fails in extremely 


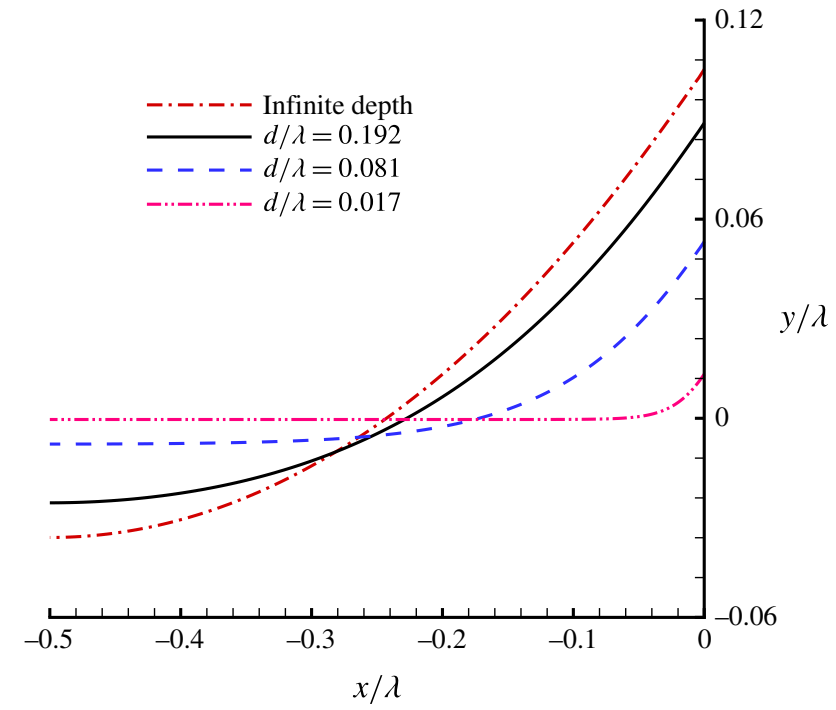

FIGURE 4. (Colour online) Wave profiles for a variety of water depths, given by the firstorder HAM-based iteration approach.

$\begin{array}{lcccc}r_{0} & \text { Schwartz (1974) } & \text { Cokelet (1977) } & \text { Williams (1981) } & \text { The HAM } \\ 0 & 1.4118 \times 10^{-1} & 1.41055 \times 10^{-1} & 1.41063 \times 10^{-1} & 1.4108 \times 10^{-1} \\ 0.1 & 1.380 \times 10^{-1} & 1.378 \times 10^{-1} & 1.37801 \times 10^{-1} & 1.3782 \times 10^{-1} \\ 0.2 & 1.285 \times 10^{-1} & 1.285 \times 10^{-1} & 1.28495 \times 10^{-1} & 1.2851 \times 10^{-1} \\ 0.3 & 1.145 \times 10^{-1} & 1.1443 \times 10^{-1} & 1.14439 \times 10^{-1} & 1.1446 \times 10^{-1} \\ 0.4 & 9.75 \times 10^{-2} & 9.739 \times 10^{-2} & 9.7374 \times 10^{-2} & 9.7388 \times 10^{-2} \\ 0.5 & 7.91 \times 10^{-2} & 7.910 \times 10^{-2} & 7.9072 \times 10^{-2} & 7.9084 \times 10^{-2} \\ 0.6 & 6.14 \times 10^{-2} & 6.090 \times 10^{-2} & 6.0984 \times 10^{-2} & 6.0995 \times 10^{-2} \\ 0.7 & 4.5 \times 10^{-2} & 4.374 \times 10^{-2} & 4.3975 \times 10^{-2} & 4.3983 \times 10^{-2} \\ 0.8 & - & 2.79 \times 10^{-2} & 2.8258 \times 10^{-2} & 2.8263 \times 10^{-2} \\ 0.9 & - & 1.5 \times 10^{-2} & 1.3667 \times 10^{-2} & 1.3670 \times 10^{-2} \\ 0.95 & - & - & - & 6.7292 \times 10^{-3} \\ 0.97 & - & - & - & 4.0128 \times 10^{-3} \\ 0.99 & - & - & - & 1.3281 \times 10^{-3}\end{array}$

TABLE 8. Limiting wave steepness, $H / \lambda$, for a variety of depths.

shallow water, i.e. $r_{0} \geqslant 0.9$. By contrast, our results given by the HAM are valid in almost arbitrary water depth. Besides, in the case of $r_{0}=0.99, H / d$ given by the HAM is in accord with the results of the highest solitary wave:

$$
\left(\frac{H}{d}\right)_{\max }=\frac{c^{2}}{2 g d} \text { for } r_{0}=1 .
$$

This suggests that the solitary wave theory could be unified into the Stokes wave theory.

According to Hedges (1995), waves with the Ursell number $H \lambda^{2} / d^{3}>4000$ are regarded as solitary waves. It is found that, in the case of $r_{0}=0.99$, corresponding 

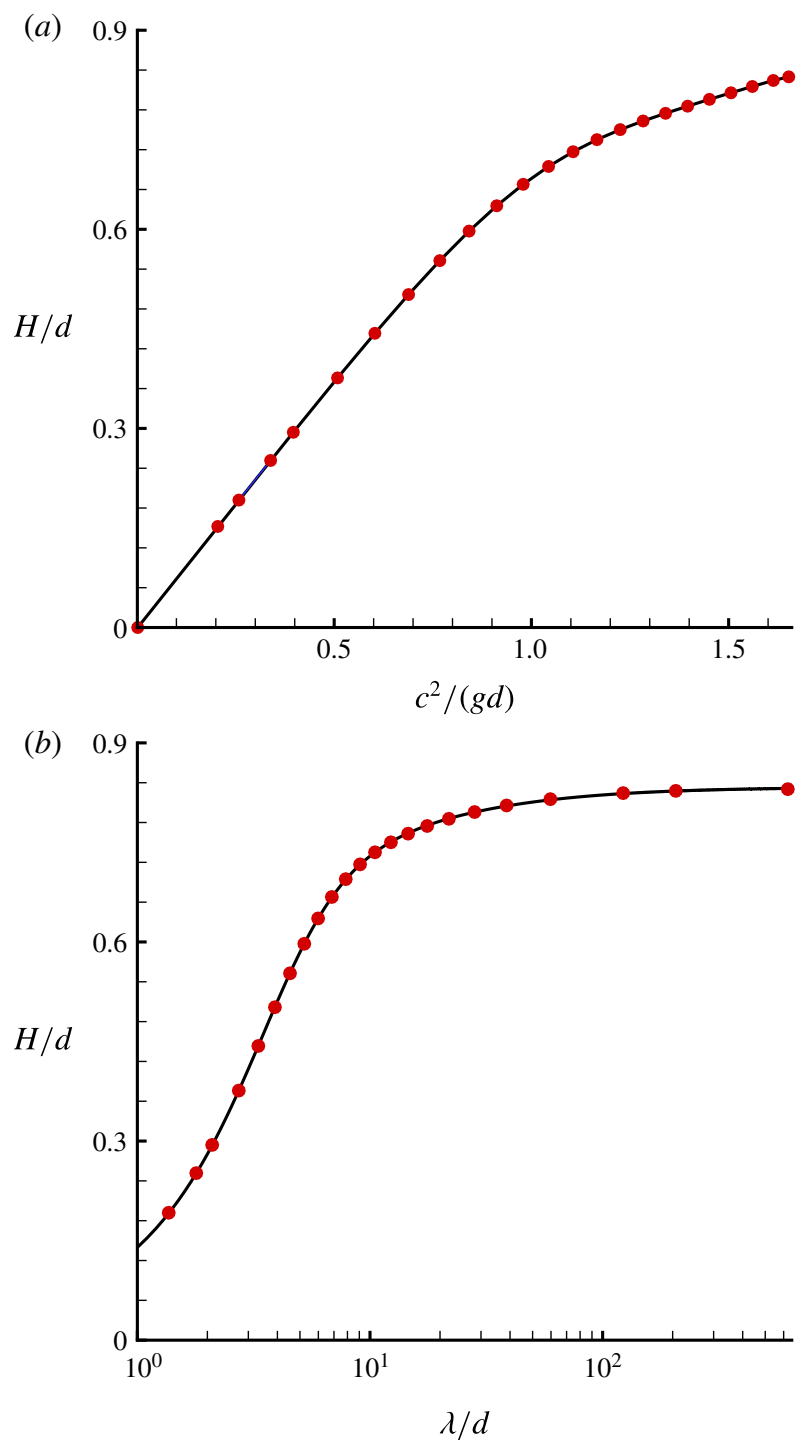

FIGURE 5. (Colour online) Comparison of $H / d$ given by the HAM-based iteration approach and fitted formulas (5.3), (5.4). @, the first-order HAM-based iteration approach; (a) $\longrightarrow$, (5.3); (b) - (5.4).

to $\lambda / d \approx 600$, the $H \lambda^{2} / d^{3}$ of the limiting Stokes wave given by the HAM reaches $3 \times 10^{5}$. Thus, the Stokes wave theory is actually valid for almost arbitrary depth, as shown in figure 8. So, in the framework of the HAM, the Stokes wave theory can describe not only the periodic waves in deep and intermediate water but also cnoidal waves in shallow water and solitary waves in extremely shallow water.

In addition, the ratio of wave height to depth, $H / d$, of the highest solitary wave has been widely studied by many researchers: $H / d=0.827$ was given by Yamada (1957), Lenau (1966), Yamada \& Shiotani (1968), Longuet-Higgins \& Fenton (1974); but $H / d=0.8332$ was given by Williams (1981), Witting (1981), Witting \& Bergin (1981), Hunter \& Vanden-Broeck (1983). Note that, $H / d=0.8303>0.827$ is given 


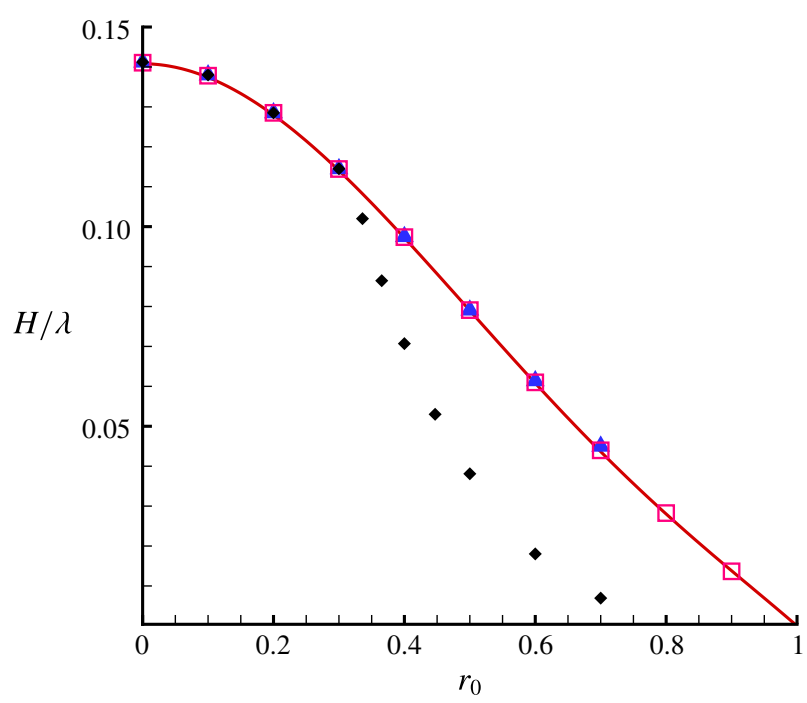

FIgure 6. (Colour online) Comparison of the limiting wave steepness $H / \lambda$.

the first-order HAM-based iteration approach; $\diamond$, perturbation method with the aid of Padé approximants (Schwartz 1974); $\boldsymbol{\Delta}$, perturbation method with the aid of both Padé approximants and Shanks' iterated $e_{1}$ transformation (Schwartz 1974); $\square$ : Williams' numerical method (Williams 1981).

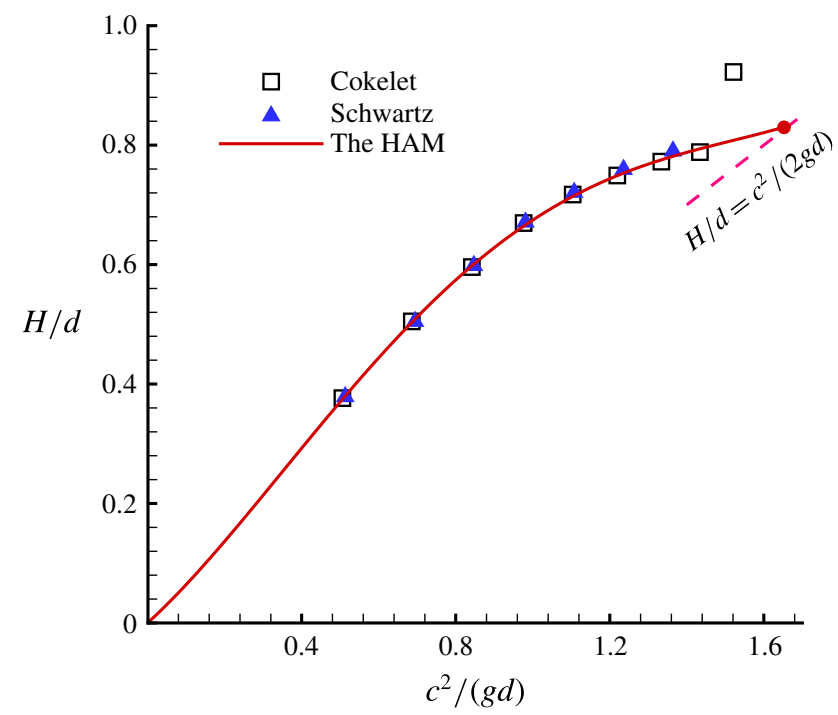

Figure 7. (Colour online) Comparison of $H / d$, versus the squared Froude number, $c^{2} /(g d)$. - , the first-order HAM-based iteration approach; $\bullet$, the case of $r_{0}=0.99$ given by the first-order HAM-based iteration approach; $\boldsymbol{\Delta}$, Schwartz (1974); $\square$, Cokelet (1977); $-\_-H / d=c^{2} /(2 g d)$.

by the HAM in the case of $r_{0}=0.99$. Hence the value $H / d=0.827$ for the highest solitary wave is denied by the HAM.

Figure 9 shows the wave profiles of the limiting wave in the case of $r_{0}=0.99$ given by the HAM from the exact wave equations, the $\mathrm{KdV}$ solution (Korteweg \& de 


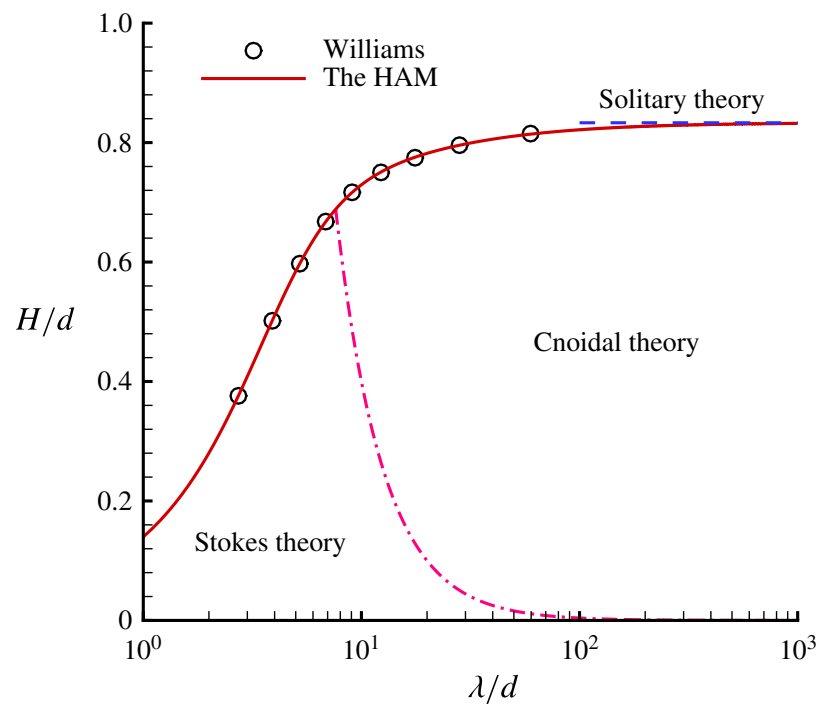

FIgURE 8. (Colour online) Comparison of $H / d$, versus $\lambda / d$. ०, Williams $(1981)$; — first-order HAM-based iteration approach; _ - - , demarcation line between Stokes and cnoidal theories, the Ursell number $H \lambda^{2} / d^{3}=40$ (Hedges 1995); ---, $(H / d)_{\max }=0.83322$ for solitary wave (Hunter \& Vanden-Broeck 1983).

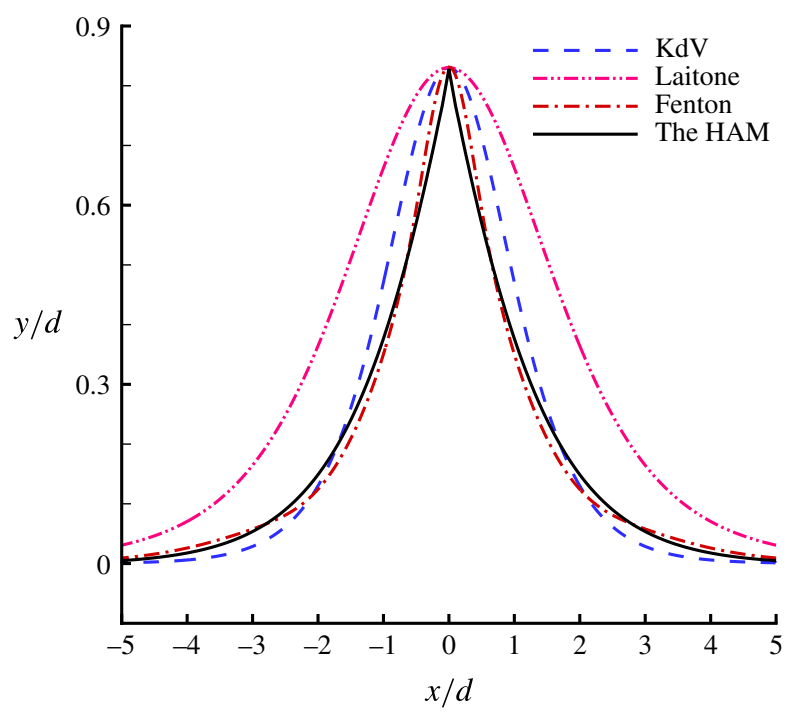

FIGURE 9. (Colour online) Wave profile in the case of $r_{0}=0.99$. ---, exact solution of KdV equation (Korteweg \& de Vries 1895); - - - Laitone's second-order approximation solution (Laitone 1960); _ - - Fenton's ninth-order approximation solution (Fenton $1972) ;-$, homotopy approximation solution of (2.10).

Vries 1895), Laitone's second-order solution (Laitone 1960) and Fenton's ninth-order solution (Fenton 1972). It is found that only the HAM gives a wave profile with a sharply pointed crest, enclosing an angle $119.2^{\circ}$. So, the KdV solution (Korteweg \& de Vries 1895), Laitone's solution (Laitone 1960) and Fenton's solution (Fenton 1972) 
are all no longer valid in the limiting case. However, compared to the famous solitary solution of the KdV equation (Korteweg \& de Vries 1895) and Laitone's solution (Laitone 1960), Fenton's ninth-order solution (Fenton 1972) is of higher accuracy.

In summary, using the iterative HAM approach with a proper convergence-control parameter, we gain limiting Stokes waves for almost arbitrary water depth, without using any extrapolation techniques. Therefore, in the framework of the HAM, the Stokes wave theory is a unified theory for all kinds of progressive waves in arbitrary depth, even including solitary waves in extremely shallow water.

\section{Concluding remarks}

Obviously, the limiting Stokes wave in shallow water is a strongly nonlinear problem. Previous methods, especially the perturbation methods, usually suffer divergence either when the wave height approaches the peak value or when the water depth is extremely small. For the limiting Stokes wave, due to the existence of a singularity located exactly at the crest, perturbation methods usually can yield convergent results only for a small part of Fourier coefficients so that the extrapolation methods such as Padé approximant techniques and Shanks' transformation have to be used.

In this paper, we employ the homotopy analysis method (HAM) to solve the limiting Stokes wave in an arbitrary depth of water. It is found that the convergence of all Fourier coefficients of the solutions can be guaranteed by choosing a proper convergence-control parameter $\hbar$ in the framework of the HAM, as shown in figure 2 . In addition, since the Fourier series is used to represent the free surface with a sharp pointed crest, using a large number of Fourier coefficients is inevitable. For other analytic/numerical methods, this might lead to rather slow convergence of the Fourier coefficients of the solutions. Fortunately, the HAM also provides us with great freedom to choose the initial guesses of solutions. Based on this kind of freedom of the HAM, we proposed an iteration HAM approach to greatly accelerate the convergence of all Fourier coefficients. Note that, since we consider a large enough number of Fourier coefficients, and more importantly, all of these coefficients are convergent without using any extrapolation methods, we can obtain the accurate wave profile even in rather shallow water.

It should be emphasized that accurate representation of the wave profile in very shallow water is impossible using other methods, especially without using any kind of extrapolation technique. For instance, although Cokelet's perturbation method (Cokelet 1977) can give results of $H / \lambda$ with acceptable accuracy for $r_{0}<0.9$, it can only give a good wave profile for $r_{0} \leqslant 0.5$. Fortunately, by means of the HAM, we gain accurate limiting wave profiles in almost arbitrary depths of water, i.e. from $r_{0}=0$ to $r_{0}=0.99$, without using any extrapolation methods such as Padé approximant techniques and Shanks' transformation. To the best of our knowledge, an accurate wave profile in the case of $r_{0}=0.99$ has been never reported. This once again illustrates the superiority of the HAM over perturbation and traditional numerical methods for this famous problem.

According to Hedges (1995), waves with the Ursell number $H \lambda^{2} / d^{3}>4000$ are regarded as solitary waves. It is found that, in the case of $r_{0}=0.99$, corresponding to $\lambda / d \approx 600$, the $H \lambda^{2} / d^{3}$ of the Stokes wave given by our HAM approach reaches $3 \times 10^{5}$. Thus, the Stokes wave theory is actually valid almost in arbitrary depth, as shown in figure 8. So in the frame of the HAM, the Stokes wave theory can describe not only the periodic waves in deep and intermediate water but also cnoidal 
waves in shallow water and solitary waves in extremely shallow water. Therefore, in the framework of the HAM, the Stokes wave is a unified theory for all kinds of progressive waves, even including the limiting (extreme) solitary waves with a sharp crest of $120^{\circ}$ angle in extremely shallow water!

Note that cubic relations between $a_{j}$ in (2.12)-(2.13) were considered in this paper, although quadratic relations between the Fourier coefficients $a_{j}$ were reported by Longuet-Higgins (1978). Certainly, the computational efficiency could be improved by means of using the quadratic relations (Longuet-Higgins 1985; Balk 1996), but one should obtain the same results as mentioned above in this paper, from a physical viewpoint.

From viewpoint of applied mathematics, this paper provides us with an additional example to illustrate that the HAM can indeed be applied to find something completely new, such as the discovery of the steady-state exactly/nearly resonant gravity waves with a time-independent wave spectrum (Liao 2011; Xu et al. 2012; Liu \& Liao 2014; Liu et al. 2015; Liao et al. 2016; Liu et al. 2018), or to attack some challenging problems with high nonlinearity.

\section{Acknowledgements}

Thanks to the anonymous reviewers for their valuable comments. Thanks to Professor Yaosong Chen (Peking University, China) for his suggesting us to attack the limiting Stokes wave in the extremely shallow water by means of the HAM. This work was carried out on TH-2 at National Supercomputer Centre in Guangzhou, China. It is partly supported by National Natural Science Foundation of China (Approval no. 11432009).

Appendix A. Detailed derivation of formulas (2.12)-(2.14)

Rewrite (2.11)

$$
f(\zeta)=\sum_{i=-r}^{r} g_{i} \zeta^{i}
$$

in which

$$
\left.\begin{array}{c}
g_{i}=a_{-i} r_{0}^{-2 i} \quad \text { when } i<0, \\
g_{0}=a_{0}, \\
g_{i}=a_{i} \quad \text { when } i>0 .
\end{array}\right\}
$$

Note that $R=1$ on the free surface, i.e. $\zeta=\mathrm{e}^{\mathrm{i} \theta}$. We have

$$
\begin{aligned}
f \bar{f} & =\left(\sum_{i=-r}^{r} g_{i} \zeta^{i}\right)\left(\sum_{i=-r}^{r} g_{i} \zeta^{-i}\right) \\
& =\sum_{i=-r}^{r} g_{i}^{2}+\sum_{k=1}^{2 r}\left[\left(\zeta^{k}+\zeta^{-k}\right)\left(\sum_{m=k-r}^{r} g_{m} g_{m-k}\right)\right] \\
& =\sum_{i=-r}^{r} g_{i}^{2}+\sum_{k=1}^{2 r}\left[2\left(\sum_{m=k-r}^{r} g_{m} g_{m-k}\right) \cos (k \theta)\right] \\
& =\sum_{k=0}^{2 r} j_{k} \cos (k \theta),
\end{aligned}
$$


where

$$
j_{0}=\sum_{i=-r}^{r} g_{i}^{2}, \quad j_{k}=2 \sum_{m=k-r}^{r} g_{m} g_{m-k}, \quad k=1,2, \ldots, 2 r .
$$

In addition, we have

$$
\begin{aligned}
\int_{0}^{\theta} \operatorname{Im}[f] \mathrm{d} \theta & =\int_{0}^{\theta}\left[\sum_{k=1}^{r} a_{k}\left(1-r_{0}^{2 k}\right) \sin (k \theta)\right] \mathrm{d} \theta \\
& =\sum_{k=1}^{r} \frac{a_{k}\left(1-r_{0}^{2 k}\right)}{k}-\sum_{k=1}^{r}\left[\frac{a_{k}\left(1-r_{0}^{2 k}\right)}{k} \cos (k \theta)\right] \\
& =\sum_{k=0}^{r} h_{k} \cos (k \theta),
\end{aligned}
$$

where

$$
h_{0}=\sum_{n_{1}=1}^{r} \frac{a_{n_{1}}\left(1-r_{0}^{2 n_{1}}\right)}{n_{1}}, \quad h_{n}=-\frac{a_{n}\left(1-r_{0}^{2 n}\right)}{n}, \quad n=1,2, \ldots, r
$$

Then we have

$$
\begin{aligned}
f \bar{f} \int_{0}^{\theta} \operatorname{Im}[f] \mathrm{d} \theta & =\left[\sum_{k=0}^{2 r} j_{k} \cos (k \theta)\right]\left[\sum_{k=0}^{r} h_{k} \cos (k \theta)\right] \\
& =\left(j_{0} h_{0}+\frac{1}{2} \sum_{n=1}^{r} j_{n} h_{n}\right)+\mathcal{N}_{1} \cos \theta+\mathcal{N}_{2} \cos (2 \theta)+\cdots,
\end{aligned}
$$

in which $\mathcal{N}_{1}, \mathcal{N}_{2}, \ldots, \mathcal{N}_{k}$ are defined by (2.13).

\section{REFERENCES}

Amick, C. J., Fraenkel, L. E. \& Toland, J. F. 1982 On the Stokes conjecture for the wave of extreme form. Acta Mathematica 148 (1), 193-214.

BALK, A. M. 1996 A lagrangian for water waves. Phys. Fluids 8 (2), 416-420.

Byatt-Smith, J. G. B. \& Longuet-Higgins, M. S. 1976 On the speed and profile of steep solitary waves. Proc. R. Soc. Lond. A 350 (1661), 175-189.

Chandler, G. A. \& Graham, I. G. 1993 The computation of water waves modelled by Nekrasov's equation. SIAM J. Numer. Anal. 30 (4), 1041-1065.

Chappelear, J. E. 1961 Direct numerical calculation of wave properties. J. Geophys. Res. 66 (2), 501-508.

Chen, B. \& Saffman, P. G. 1980 Numerical evidence for the existence of new types of gravity waves of permanent form on deep water. Stud. Appl. Maths 62, 1-21.

Cokelet, E. D. 1977 Steep gravity waves in water of arbitrary uniform depth. Phil. Trans. R. Soc. Lond. 286 (1335), 183-230.

Crew, S. C. \& Trinh, P. H. 2016 New singularities for Stokes waves. J. Fluid Mech. 798, 256-283.

Dallaston, M. C. \& Mccue, S. W. 2010 Accurate series solutions for gravity-driven Stokes waves. Phys. Fluids 22 (8), p082104. 
Davies, T. V. 1951 The theory of symmetrical gravity waves of finite amplitude. I. Proc. R. Soc. Lond. A 208 (1095), 475-486.

DEAN, R. G. 1965 Stream function representation of nonlinear ocean waves. J. Geophys. Res. 70 (18), 4561-4572.

Dyachenko, S. A., Lushnikov, P. M. \& Korotkevich, A. O. 2014 Complex singularity of a Stokes wave. J. Expl Theor. Phys. Lett. 98 (11), 675-679.

Dyachenko, S. A., Lushnikov, P. M. \& KorotKevich, A. O. 2016 Branch cuts of Stokes wave on deep water. Part I: numerical solution and Padé approximation. Stud. Appl. Maths 137 (4), 419-472.

Fenton, J. D. 1972 A ninth-order solution for the solitary wave. J. Fluid Mech. 53 (2), 257-271.

Fenton, J. D. 1988 The numerical solution of steady water wave problem. Comput. Geosci. 14 (3), 357-368.

Fenton, J. D. 1990 Nonlinear wave theories. Ocean Engng Sci. 9, 1-18.

GRAnt, M. A. 1973 The singularity at the crest of a finite amplitude progressive Stokes wave. J. Fluid Mech. 59 (2), 257-262.

Hedges, T. S. 1995 Regions of validity of analytical wave theories. Ice Proceedings Water Maritime Energy 112 (2), 111-114.

Hunter, J. K. \& VAnden-Broeck, J. M. 1983 Accurate computations for steep solitary waves. J. Fluid Mech. 136 (136), 63-71.

KARABUT, E. A. 1998 An approximation for the highest gravity waves on water of finite depth. J. Fluid Mech. 372, 45-70.

Kimiaeifar, A., Lund, E., Thomsen, O. T. \& Srensen, J. D. 2011 Application of the homotopy analysis method to determine the analytical limit state functions and reliability index for large deflection of a cantilever beam subjected to static co-planar loading. Comput. Maths Applics. 62 (12), 4646-4655.

Klopman, G. 1990 A note on integral properties of periodic gravity waves in the case of a non-zero mean Eulerian velocity. J. Fluid Mech. 211 (5), 609-615.

KorteweG, D. J. \& DE VRIES, G. 1895 On the change of form of long waves advancing in a rectangular canal and on a new type of long stationary waves. Phil. Mag. 39, 422-443.

LAitone, E. V. 1960 The second approximation to cnoidal and solitary waves. J. Fluid Mech. 9 (3), 430-444.

Lenau, C. W. 1966 The solitary wave of maximum amplitude. J. Fluid Mech. 26 (2), 309-320.

LIAO, S. J. 1992 Proposed homotopy analysis techniques for the solution of nonlinear problem. PhD thesis, Shanghai Jiao Tong University.

LIAO, S. J. 1999 A uniformly valid analytic solution of 2D viscous flow past a semi-infinite flat plate. J. Fluid Mech. 385, 101-128.

LiAO, S. J. 2003 Beyond Perturbation: Introduction to Homotopy Analysis Method. Chapman \& Hall/CRC.

LiaO, S. J. 2009 Notes on the homotopy analysis method: some definitions and theorems. Commun. Nonlinear Sci. Numer. Simul. 14 (4), 983-997.

LiAO, S. J. 2010 An optimal homotopy-analysis approach for strongly nonlinear differential equations. Commun. Nonlinear Sci. Numer. Simul. 15 (8), 2003-2016.

LiaO, S. J. 2011 On the homotopy multiple-variable method and its applications in the interactions of nonlinear gravity waves. Commun. Nonlinear Sci. Numer. Simul. 16 (3), 1274-1303.

LiaO, S. J. 2012 Homotopy Analysis Method in Nonlinear Differential Equations. Springer.

Liao, S. J., XU, D. L. \& Stiassnie, M. 2016 On the steady-state nearly resonant waves. J. Fluid Mech. 794, 175-199.

LIU, Z. \& LiaO, S. J. 2014 Steady-state resonance of multiple wave interactions in deep water. J. Fluid Mech. 742, 664-700.

Liu, Z., Xu, D. L., Li, J., Peng, T., Alsaedi, A. \& Liao, S. J. 2015 On the existence of steady-state resonant waves in experiments. J. Fluid Mech. 763, 1-23.

LiU, Z., XU, D. L. \& LiaO, S. J. 2017 Mass, momentum and energy flux conservation between linear and nonlinear steady-state wave groups. Phys. Fluids 29, 127104. 
LiU, Z., XU, D. L. \& LiaO, S. J. 2018 Finite amplitude steady-state wave groups with multiple near resonances in deep water. J. Fluid Mech. 835, 624-653.

Longuet-Higgins, M. S. 1975 Integral properties of periodic gravity waves of finite amplitude. Proc. R. Soc. Lond. 342 (1629), 157-174.

Longuet-Higgins, M. S. 1978 Some new relations between Stokes's coefficients in the theory of gravity waves. IMA J. Appl. Maths 22 (3), 261-273.

Longuet-Higgins, M. S. 1985 Bifurcation in gravity waves. J. Fluid Mech. 151, 457-475.

Longuet-Higgins, M. S. \& Fenton, J. D. 1974 On the mass, momentum, energy and circulation of a solitary wave. II. Proc. R. Soc. Lond. A A340, 471-493.

Longuet-Higgins, M. S. \& Fox, M. J. H. 1978 Theory of the almost-highest wave. Part 2. Matching and analytic extension. J. Fluid Mech. 85 (4), 769-786.

Lushnikov, P. M. 2016 Structure and location of branch point singularities for Stokes waves on deep water. J. Fluid Mech. 800, 557-594.

Lushnikov, P. M., Dyachenko, S. A. \& Silantyev, D. A. 2017 New conformal mapping for adaptive resolving of the complex singularities of Stokes wave. Proc. R. Soc. Lond. A 473, 20170198; doi:10.1098/rspa.2017.0198.

Mastroberardino, A. 2011 Homotopy analysis method applied to electrohydrodynamic flow. Commun. Nonlinear Sci. Numer. Simul. 16 (7), 2730-2736.

Michell, J. H. 1893 The highest waves in water. Phil. Mag. 36 (5), 430-437.

Nekrasov, A. I. 1920 On Stokes' wave. Isv. Ivanovo-Voznesesk. Politekhn 81-89.

Olfe, D. B. \& Rottman, J. W. 1980 Some new highest-wave solutions for deep-water waves of permanent form. J. Fluid Mech. 100 (4), 801-810.

Plotnikov, P. I. 2002 A proof of the Stokes conjecture in the theory of surface waves*. Stud. Appl. Maths 108 (2), 217-244; translated from Dinamika Sploshn. Sredy 57 (1982) 41-76.

Rienecker, M. M. \& Fenton, J. D. 1981 A Fourier approximation method for steady water waves. J. Fluid Mech. 104 (104), 119-137.

Sardanyés, J., Rodrigues, C., Januário, C., Martins, N., Gil-Gómez, G. \& Duarte, J. 2015 Activation of effector immune cells promotes tumor stochastic extinction: a homotopy analysis approach. Appl. Math. Comput. 252, 484-495.

Schwartz, L. W. 1972 Analytic continuation of Stokes' expansion for gravity waves. PhD thesis, Stanford University.

SChWARTZ, L. W. 1974 Computer extension and analytic continuation of Stokes' expansion for gravity waves. J. Fluid Mech. 62 (3), 553-578.

Schwartz, L. W. \& Fenton, J. D. 1982 Strongly nonlinear waves. Annu. Rev. Fluid Mech. 14, 39-60.

SHANKS, D. 1954 Non-linear transformations of divergent and slowly convergent sequences. J. Math. Phys. 34 (1), 1-42.

Stokes, G. G. 1847 On the theory of oscillatory waves. Trans. Camb. Phil. Soc. 8, 441-455.

Stokes, G. G. 1880 Supplement to a paper on the theory of oscillatory waves. Math. Phys. Papers (1), 314-326.

Sulem, C., Sulem, P. L. \& Frisch, H. 1983 Tracing complex singularities with spectral methods. J. Comput. Phys. 50 (1), 138-161.

Tanveer, S. 1991 Singularities in water waves and Rayleigh-Taylor instability. Proc. R. Soc. Lond. A 435 (1893), 137-158.

Tulin, M. P. 1983 An exact theory of gravity wave generation by moving bodies, its approximation and its implication. In 14th Symposium on Naval Hydrodynamics, pp. 19-51. Academic Press.

Vajravelu, K. \& Van Gorder, R. A. 2012 Nonlinear Flow Phenomena and Homotopy Analysis: Fluid Flow and Heat Transfer. Springer.

Van Gorder, R. A. \& Vajravelu, K. 2008 Analytic and numerical solutions to the Lane-Emden equation. Phys. Lett. A 372, 6060-6065.

VAnden-Broeck, J. M. 1986 Steep gravity waves: Havelock method revisited. Phys. Fluids 29 (9), 3084-3085. 
Vanden-Broeck, J. M. \& Schwartz, L. W. 1979 Numerical computation of steep gravity waves in shallow water. Phys. Fluids 22 (10), 1868-1871.

Vanden-Broeck, J. M. \& Miloh, T. 1995 Computations of steep gravity waves by a refinement of DaviesTulins approximation. SIAM J. Appl. Maths 55 (4), 892-903.

Williams, J. M. 1981 Limiting gravity waves in water of finite depth. Phil. Trans. R. Soc. A 302 (1466), 139-188.

Witting, J. M. 1981 High solitary waves in water: results of calculations. Naval Research Lab Report 8505.

Witting, J. M. \& Bergin, J. M. 1981 High solitary waves in water: a refined numerical method. Naval Research Lab Report 8504.

Xu, D. L., Lin, Z. L., LiaO, S. J. \& Stiassnie, M. 2012 On the steady-state fully resonant progressive waves in water of finite depth. J. Fluid Mech. 710, 379-418.

YamadA, H. 1957 Highest waves of permanent type on the surface of deep water. Appl. Mech. Res. Rep., Kyushu Univ. 5 (18), 37-52.

YAmada, H. \& Shiotani, T. 1968 On the highest water waves of permanent type. Bull. Disas. Prey. Res. Inst. 18 (2), 1-22.

Zhong, X. X. \& LiaO, S. J. 2017 Analytic solutions of Von Kármán plate under arbitrary uniform pressure - equations in differential form. Stud. Appl. Maths 138, 371-400.

Zhong, X. X. \& LiaO, S. J. 2018 Analytic solutions of Von Kármán plate under arbitrary uniform pressure - equations in integral form. Sci. China Phys. Mech. Astronomy 61, 014611. 